\title{
Randomized trial of preoperative docetaxel with or without capecitabine after 4 cycles of 5-fluorouracil- epirubicin-cyclophosphamide (FEC) in early-stage breast cancer: exploratory analyses identify Ki67 as a predictive biomarker for response to neoadjuvant chemotherapy
}

\author{
S. Ohno $\cdot$ L. W. C. Chow $\cdot$ N. Sato $\cdot$ N. Masuda $\cdot$ H. Sasano $\cdot$ F. Takahashi $\cdot$ H. Bando $\cdot$ \\ H. Iwata - T. Morimoto - S. Kamigaki - T. Nakayama - S. Nakamura • K. Kuroi • \\ K. Aogi • M. Kashiwaba • H. Yamashita • K. Hisamatsu • Y. Ito • Y. Yamamoto • \\ T. Ueno $\cdot$ E. Fakhrejahani $\cdot$ N. Yoshida $\cdot$ M. Toi
}

Received: 24 May 2013/ Accepted: 29 August 2013/Published online: 12 October 2013

(C) The Author(s) 2013. This article is published with open access at Springerlink.com

\begin{abstract}
This randomized, multicenter study compared the efficacy of docetaxel with or without capecitabine following fluorouracil/epirubicin/cyclophosphamide (FEC) therapy in operable breast cancer and investigated the role of Ki67 as a predictive biomarker. Patients were randomized to 4 cycles of docetaxel/capecitabine (docetaxel: $75 \mathrm{mg} / \mathrm{m}^{2}$ on day 1 ; capecitabine: $1,650 \mathrm{mg} / \mathrm{m}^{2}$ on days $1-14$ every 3 weeks) or docetaxel alone $\left(75 \mathrm{mg} / \mathrm{m}^{2}\right.$ on day 1 every 3 weeks) after completion of 4 cycles of FEC (5-fluorouracil $500 \mathrm{mg} / \mathrm{m}^{2}$, epirubicin $100 \mathrm{mg} / \mathrm{m}^{2}$ and cyclophosphamide $500 \mathrm{mg} / \mathrm{m}^{2}$ on day 1 every 3 weeks). The primary endpoint was the pathological complete response
\end{abstract}

Electronic supplementary material The online version of this article (doi:10.1007/s10549-013-2691-y) contains supplementary material, which is available to authorized users.

\section{S. Ohno}

Division of Clinical Oncology, National Kyushu Cancer Center, Fukuoka, Japan

S. Ohno $(\bowtie)$

OOTR Institute, $1 \mathrm{~F}$, Kyoto Technoscience Center,

14 Yoshida-Kawara-cho, Sakyo-ku, Kyoto 606-8305, Japan

e-mail: info@ootr-institute.org

\section{W. C. Chow}

Unimed Medical Institute, Wanchai, Hong Kong

\section{N. Sato}

Department of Surgery, Niigata Cancer Center Hospital, Niigata, Japan

N. Masuda

Breast Oncology Unit, Department of Surgery, Osaka National

Hospital, Osaka, Japan
(pCR) rate. Predictive factor analysis was conducted using clinicopathological markers, including hormone receptors and Ki67 labeling index (Ki67LI). A total of 477 patients were randomized; the overall response in the docetaxel/ capecitabine and docetaxel groups was 88.3 and $87.4 \%$, respectively. There were no significant differences in the pCR rate (docetaxel/capecitabine: $23 \%$; docetaxel: $24 \%$; $p=0.748)$, disease-free survival, or overall survival. However, patients with mid-range Ki67LI (10-20\%) showed a trend towards improved pCR rate with docetaxel/ capecitabine compared to docetaxel alone. Furthermore, multivariate logistic regression analysis showed pre-treatment Ki67LI (odds ratio 1.031; $95 \%$ CI 1.014-1.048; $p=0.0004)$ to be a significant predictor of $\mathrm{pCR}$ in this neoadjuvant treatment setting. Docetaxel/capecitabine

\section{H. Sasano}

Department of Pathology, Tohoku University School of Medicine, Sendai, Japan

F. Takahashi

Department of Clinical Medicine (Biostatistics), School of

Pharmacy, Kitasato University, Tokyo, Japan

\section{H. Bando}

Department of Breast and Endocrine Surgery, Graduate School of Comprehensive Human Sciences, University of Tsukuba, Tsukuba, Japan

H. Iwata

Department of Breast Oncology, Aichi Cancer Center Hospital, Nagoya, Japan 
(after 4 cycles of FEC) did not generate significant improvement in pCR compared to docetaxel alone. However, exploratory analyses suggested that assessment of pre-treatment Ki67LI may be a useful tool in the identification of responders to preoperative docetaxel/capecitabine in early-stage breast cancer.

Keywords Breast cancer - Neoadjuvant chemotherapy · Ki67 - Capecitabine · Pathological complete response $\cdot$ Docetaxel

\section{Introduction}

Neoadjuvant chemotherapy has become increasingly significant in the treatment of operable early-stage breast cancer, with the advantage of the potential to downgrade tumors and increase the rate of breast conserving surgery (BCS) in patients that may have otherwise required a mastectomy [1]. Results from the National Surgical Adjuvant Breast and Bowel Project (NSABP) protocol B-18 trial demonstrated an increased likelihood in BCS in breast cancer patients treated with a neoadjuvant anthracycline-based regimen [1]. Although the B-18 trial did not demonstrate a survival advantage in patients treated with preoperative chemotherapy, it established pathological complete response (pCR) as a prognostic marker for disease-free survival (DFS). Indeed, pCR after neoadjuvant chemotherapy is considered a marker for favorable prognosis in breast cancer patients [2].

As such, clinical and molecular biomarkers capable of predicting $\mathrm{pCR}$ have been assessed following neoadjuvant treatment in breast cancer patients $[3,4]$. In particular, the

\section{T. Morimoto}

Department of Breast Surgery, Yao Municipal Hospital, Yao, Japan

S. Kamigaki · T. Nakayama

Department of Mammary Gland and Internal Secretion Surgery, Sakai City Hospital, Sakai, Japan

S. Nakamura

Department of Breast Surgical Oncology, St.Luke's International

Hospital, Tokyo, Japan

K. Kuroi

Division of Clinical Trials and Research, Department of Surgery, Tokyo Metropolitan Komagome Hospital, Tokyo, Japan

K. Aogi

Department of Breast Oncology, NHO Shikoku Cancer Center, Matsuyama, Japan

M. Kashiwaba

Department of Surgery, Iwate Medical University, Morioka,

Japan proliferation marker $\mathrm{Ki} 67$ has been reported to have predictive and prognostic value in patients with invasive breast cancer who received a range of neoadjuvant chemotherapy regimens, including anthracycline-based regimen without taxanes and anthracycline and taxane-based protocols [5].

While neoadjuvant treatment with anthracycline-based regimens is highly effective in the treatment of breast cancer, the sequential addition of a taxane to an anthracycline-based neoadjuvant regimen has been demonstrated to induce additive efficacy. In the NSABP B-27 trial, the sequential addition of docetaxel after doxorubicin and cyclophosphamide (AC) therapy doubled the rate of $\mathrm{pCR}$, increased clinical response and increased the proportion of negative axillary nodes in early breast cancer patients [6]. In addition, 5-fluorouracil-epirubicin and cyclophosphamide (FEC) followed by docetaxel as neoadjuvant chemotherapy in the Japan Breast Cancer Research Group (JBCRG) 01 trial resulted in a pCR rate of $16 \%$ with BCS possible for $85 \%$ of the patients assessed [7].

In addition to inducing increased efficacy with anthracyclines, docetaxel has demonstrated significant synergy with the oral prodrug capecitabine [8]. Capecitabine is converted to 5-fluorouracil in a three-step process catalyzed by thymidine phosphorylase (TP) [9] and exhibits tumor specificity by exploiting the significantly higher activity of TP in tumor tissue in comparison to healthy tissue [8, 9]. Docetaxel has been demonstrated to upregulate TP expression in tumor tissues, possibly accounting for the synergistic effect observed with capecitabine [8]. Clinical studies have shown that single-agent capecitabine was an active and tolerable treatment for metastatic breast cancer (MBC) with disease progression during and after anthracycline and

H. Yamashita

Department of Breast and Endocrine Surgery, Nagoya City

University Hospital, Nagoya, Japan

K. Hisamatsu

Department of Surgery, Hiroshima City Asa Hospital,

Hiroshima, Japan

Y. Ito

Department of Surgery, Higashi Municipal Hospital, Nagoya, Japan

Y. Yamamoto

Department of Breast and Endocrine Surgery, Kumamoto

University Hospital, Kumamoto, Japan

T. Ueno · E. Fakhrejahani · N. Yoshida · M. Toi

Breast Surgery Department, Kyoto University Hospital, Kyoto, Japan 
taxane therapy, achieving response rates of 20-29\% and a median survival in excess of 1 year [10,11].

On the basis of these findings, the docetaxel/capecitabine regimen has been demonstrated to be well tolerated and effective for neoadjuvant treatment of stage II/III or locally advanced breast cancer [12-14]. Another study by O'Shaugnessy and colleagues also demonstrated a superior clinical response and survival outcome when the docetaxel/ capecitabine regimen was compared with docetaxel alone in women with anthracycline-pretreated MBC [15]. However, these studies [12-15] did not undertake analyses to identify the tumor characteristics that define patients likely to respond to neoadjuvant docetaxel/capecitabine treatment.

Our randomized trial compared the efficacy of preoperative FEC followed by docetaxel with or without capecitabine in patients with early-stage breast cancer and assessed biomarkers that may be used to identify responders, in order to establish individualized treatment regimens.

\section{Patients and methods}

\section{Study design}

This multicenter, randomized, open study compared the efficacy of 4 cycles of FEC followed by 4 cycles of docetaxel and capecitabine or 4 cycles of docetaxel alone as neoadjuvant chemotherapy in patients with operable breast cancer. The study was approved by the Institutional Review Board of the Organisation of Oncology and Translational Research and conducted according to the Declaration of Helsinki. The primary endpoint was the pCR rate; secondary endpoints included toxicity, clinical response, frequency of breast and axillary lymph node conservation surgery, DFS, and overall survival (OS).

\section{Patient eligibility}

Women (20-70 years) with histologically confirmed operable invasive breast adenocarcinoma (T1C-3, N0, M0 $(>1 \mathrm{~cm}) / \mathrm{T} 1-3, \mathrm{~N} 1, \mathrm{M} 0)$ were eligible. In women without clinically suspicious axillary adenopathy, the primary breast tumor had to be $>1 \mathrm{~cm}$ in diameter; patients with clinically suspicious axillary adenopathy could present with a primary tumor of any size (in accordance with cancer staging as per the American Joint Committee on Cancer).

Inclusion criteria were as follows: no prior treatment for breast cancer, Eastern Cooperative Oncology Group performance status of $0-1$, white blood cell count $>4,000-12,000 \mathrm{~mm}^{3}$ or neutrophil count $>2,000 \mathrm{~mm}^{3}$, platelets $>100,000 \mathrm{~mm}^{3}$, hemoglobin $>9.5 \mathrm{~g} / \mathrm{dL}$, bilirubin $<1.25 \times$ institutional upper limit of normal (ULN), creatinine $<1.5 \times$ institutional ULN, creatinine clearance $>30 \mathrm{~mL} /$ min, aspartate aminotransferase and alanine aminotransferase $<1.5 \times$ institutional ULN, a normal electrocardiogram for cardiac function, and left ventricular ejection fraction of $>60 \%$.

Exclusion criteria included uncontrolled medical conditions, significant interstitial pneumonia or pulmonary fibrosis, suspected of infection with fever, symptomatic varicella, required treatment for pleural or pericardial effusions, severe edema, severe peripheral neuropathy, required steroid pre-treatment, severe psychiatric disorders, inflammatory breast cancer, bilateral cancer (if both tumors were within the inclusion criteria, bilateral cancer was not excluded), and a history of other malignancies within the last 5 years (except for adequately treated non-melanoma skin cancer or carcinoma in situ of the cervix).

Study treatment

Patients were scheduled to receive 4 cycles of intravenous FEC (5-fluorouracil $500 \mathrm{mg} / \mathrm{m}^{2}$, epirubicin $100 \mathrm{mg} / \mathrm{m}^{2}$, cyclophosphamide $500 \mathrm{mg} / \mathrm{m}^{2}$ ) on day 1 every 3 weeks. Patients who completed 4 FEC cycles were randomly assigned to receive either 4 cycles of docetaxel $\left(75 \mathrm{mg} / \mathrm{m}^{2}\right.$, on day 1) plus capecitabine $\left(825 \mathrm{mg} / \mathrm{m}^{2}\right.$ twice daily on days $1-14)$ or 4 cycles of docetaxel alone $\left(75 \mathrm{mg} / \mathrm{m}^{2}\right.$, on day 1$)$ every 3 weeks. For patients with a creatinine clearance of $30-50 \mathrm{~mL} / \mathrm{min}$, the initial dose of capecitabine was reduced to $75 \%$ of the planned dose. Patients with disease progression while on FEC were excluded from randomization. A maximum $25 \%$ dose reduction and 3-week dose delay were permitted for adverse events. Whereas a $75 \%$ dose level was used as the initial dose for patients with low creatinine clearance, a further $25 \%$ dose reduction was permitted for adverse events. Treatment prior to docetaxel comprised dexamethasone $(8 \mathrm{mg}$ oral; administered the morning and night before docetaxel). In addition, dexamethasone (10 $\mathrm{mg}$ intravenous) was administered $30 \mathrm{~min}$ before docetaxel. If a patient missed the $8 \mathrm{mg}$ oral dexamethasone, the $10 \mathrm{mg}$ intravenous dose was still administered and docetaxel administration occurred as planned. Primary surgery was undertaken within 3-6 weeks of neoadjuvant chemotherapy completion. Supportive care and postoperative endocrine or radiation therapy were administered at the investigator's discretion. No patients received trastuzumab before surgery, as it was not approved in Japan at the time of the study.

Study assessments

Pre-enrolment assessments included medical history, physical examination, blood chemistry, bilateral mammogram, bone and computed tomography scans. Initial diagnosis of invasive adenocarcinoma was made by core needle biopsy. 
Estrogen receptor (ER) and progesterone receptor $(\mathrm{PgR})$ status were confirmed by immunohistochemistry (IHC) before randomization. Human epidermal growth factor receptor 2 (HER2) status was confirmed by IHC or fluorescent in situ hybridization. For biomarker analysis, IHC was undertaken using a mouse anti-human TP monoclonal antibody (Chugai Pharmaceutical Co., Japan). TP immunoreactivity was detected in the cytoplasm of carcinoma cells and semi-quantitative evaluation was undertaken using $>1,000$ carcinoma cells in each case. Ki67 immunostaining was performed using MIB1 monoclonal antibody (Dako Co.Ltd.) as previously described [16]. Briefly, Ki67 was stained after overnight preparation using a 1:100 dilution of the antibody. Evaluation of Ki67 was performed by counting $\geq 1,000$ carcinoma cells from each patient in the hot spots and the percentage of immunoreactivity was subsequently determined by a labelling index [17].

Clinicopathological assessments were undertaken at the central laboratory (Department of Anatomic Pathology, Tohoku University, Graduate School of Medicine, Japan). The clinical response was evaluated in accordance with the Response Evaluation Criteria In Solid Tumors guidelines. Tumor response evaluation was performed after cycles 4 and 8 , and after each cycle where possible pCR was defined as no histological evidence of invasive carcinoma, or the appearance of only non-invasive or in situ carcinoma on pathologic examination of the surgical specimen. When histological diagnosis of pCR was difficult based on hematoxylin-eosin-stained tissue sections, irrespective of whether carcinoma cells were present as ductal carcinoma in situ components, immunohistochemistry of myoepithelial markers such as cytokeratin 5/6 and p63 was used to determine the presence of invasive carcinoma [18-20]. Toxicity was graded and reported according to the NCI Common Terminology Criteria for Adverse Events version 3.

\section{Statistical analysis}

Following a reported $16 \%$ pCR rate when FEC was followed by docetaxel alone in the JBCRG 01 trial [7], it was determined that 434 assessable patients were required for randomization to achieve $80 \%$ power for the detection of an increase in the proportion of pCR rate of the docetaxel/ capecitabine versus docetaxel group. Differences in pCR rates were calculated using a one-sided Chi square test with Schouten collection at the alpha level of $5 \%$; $95 \%$ confidence interval (CI) was also calculated. In predictive factor analysis, the interaction of pCR with Ki67 as a continuous variable was explored using the subpopulation treatment effect pattern plots (STEPP) method. For each risk factor, the odds ratio (OR) for $\mathrm{pCR}$ and $95 \% \mathrm{CI}$ was calculated using simple and multivariate logistic regression models. DFS and OS were calculated using the KaplanMeier method. For each prognostic factor, hazard ratio (HR) for DFS and $95 \%$ CI was calculated using the simple Cox model. Factors associated with DFS in univariate analysis were included in the multivariate Cox model.

\section{Results}

\section{Patient population}

A total of 504 patients were enrolled into the study ( 15 centers in Japan, 1 in China, and 1 in Hong Kong), 27 of whom withdrew during FEC therapy. Following FEC therapy, 239 patients were randomly assigned to the docetaxel/ capecitabine group and 238 patients to the docetaxel alone group; all 477 patients were included in the intent-to-treat (ITT) population. Patients randomized to both groups were well balanced with respect to age, menopausal status, and baseline tumor characteristics (Table 1).

\section{Treatment administration and study completion}

No significant differences were observed in the delivery of FEC therapy between the treatment groups. However, the relative dose intensities for docetaxel were significantly lower in the docetaxel/capecitabine group than in the docetaxel alone group $(p=0.0006)$. A $25 \%$ dose reduction was required for $33 \%(79 / 239)$ of patients in the docetaxel/capecitabine group and $5.9 \%$ (14/238) of patients in the docetaxel alone group. The rate of completion after the initial dose was significantly lower in the docetaxel/capecitabine group compared with the docetaxel alone group (44.8 and $88.7 \%$, respectively; $p<0.0001$ ). Study discontinuation was significantly higher in the docetaxel/capecitabine $(53 / 239 ; 22 \%)$ group compared to docetaxel alone $(13 / 238,5.5 \% ; p<0.0001)$. The majority of study withdrawals were attributed to drug toxicity (docetaxel/capecitabine: 31/53 patient; docetaxel alone: 9/13 patients; Fig. 1).

\section{Clinical and pathological response}

The overall response rate (cCR and cPR) was $88.3 \%$ (211/ $239)$ in the docetaxel/capecitabine group and $87.4 \%$ (208/ 238 ) in the docetaxel group; no significant differences in clinical response were noted. The proportion of BCS was $70.7 \%(169 / 239)$ in the docetaxel/capecitabine group and $71.4 \%(170 / 238)$ in the docetaxel group; the proportion of axillary lymph node conservation surgery was $28.9 \%$ (69/ 239 ) and $27.7 \%$ (66/238), respectively (data not shown).

The pCR rate was $23 \%$ in the docetaxel/capecitabine group and $24 \%$ in the docetaxel group $(p=0.748$; 
Table 1 Baseline patient demographics and clinical characteristics

\begin{tabular}{|c|c|c|c|c|c|}
\hline Number & $\begin{array}{l}\text { Total } \\
504\end{array}$ & $\begin{array}{l}\text { FEC only } \\
27\end{array}$ & $\begin{array}{l}\mathrm{FEC}+\mathrm{T} \\
238\end{array}$ & $\begin{array}{l}\mathrm{FEC}+\mathrm{TX} \\
239\end{array}$ & $p$ value \\
\hline \multicolumn{6}{|l|}{ Age } \\
\hline Median & 49.0 & 47.0 & 49.0 & 49.0 & \multirow[t]{2}{*}{ W:0.8769 } \\
\hline Range & $25.0-70.0$ & $28.0-65.0$ & $25.0-68.0$ & $25.0-70.0$ & \\
\hline \multicolumn{6}{|l|}{ Menopausal status } \\
\hline Premenopausal & $282(56.0 \%)$ & $16(59.3 \%)$ & $133(55.9 \%)$ & $133(55.6 \%)$ & \multirow[t]{2}{*}{ C:0.9590 } \\
\hline Postmenopausal & $222(44.0 \%)$ & $11(40.7 \%)$ & $105(44.1 \%)$ & $106(44.4 \%)$ & \\
\hline \multicolumn{6}{|l|}{ Initial tumor size } \\
\hline Median & 3.5 & 3.5 & 3.5 & 3.5 & \multirow[t]{2}{*}{ W:0.7508 } \\
\hline Range & $0.8-10.5$ & $2.0-10.5$ & $0.8-8.0$ & $1.0-9.0$ & \\
\hline \multicolumn{6}{|l|}{ Axillary lymph nodes* } \\
\hline Positive & $280(55.6 \%)$ & $12(44.4 \%)$ & $134(56.3 \%)$ & $134(56.1 \%)$ & \multirow[t]{2}{*}{ C:0.9586 } \\
\hline Negative & $224(44.4 \%)$ & $15(55.6 \%)$ & $104(43.7 \%)$ & $105(43.9 \%)$ & \\
\hline \multicolumn{6}{|l|}{ Clinical stage } \\
\hline I & $5(1.0 \%)$ & $0(0.0 \%)$ & $2(0.8 \%)$ & $3(1.3 \%)$ & \multirow[t]{4}{*}{ C: 0.9170} \\
\hline IIA & $218(43.3 \%)$ & $12(44.4 \%)$ & $100(42.0 \%)$ & $106(44.4 \%)$ & \\
\hline IIB & $226(44.8 \%)$ & $11(40.7 \%)$ & $110(46.2 \%)$ & $105(43.9 \%)$ & \\
\hline IIIA & $55(10.9 \%)$ & $4(14.8 \%)$ & $26(10.9 \%)$ & $25(10.5 \%)$ & \\
\hline \multicolumn{6}{|l|}{ Histologic type } \\
\hline Infiltrating ductal carcinoma & $491(97.4 \%)$ & $25(92.6 \%)$ & $233(97.9 \%)$ & $233(97.5 \%)$ & \multirow[t]{6}{*}{ C:0.1087 } \\
\hline Infiltrating lobular carcinoma & $8(1.6 \%)$ & $1(3.7 \%)$ & $1(0.4 \%)$ & $6(2.5 \%)$ & \\
\hline Mucinous carcinoma & $1(0.2 \%)$ & $0(0.0 \%)$ & $1(0.4 \%)$ & $0(0.0 \%)$ & \\
\hline Invasive micropapillary carcinoma & $1(0.2 \%)$ & $0(0.0 \%)$ & $1(0.4 \%)$ & $0(0.0 \%)$ & \\
\hline Infiltrated apocrine carcinoma & $2(0.4 \%)$ & $0(0.0 \%)$ & $2(0.8 \%)$ & $0(0.0 \%)$ & \\
\hline Invasive small cell carcinoma & $1(0.2 \%)$ & $1(3.7 \%)$ & $0(0.0 \%)$ & $0(0.0 \%)$ & \\
\hline \multicolumn{6}{|l|}{ Histologic type } \\
\hline Infiltrating ductal carcinoma & $491(97.4 \%)$ & $25(92.6 \%)$ & $233(97.9 \%)$ & $233(97.5 \%)$ & \multirow[t]{2}{*}{ C:0.7657 } \\
\hline Otherwise & $13(2.6 \%)$ & $2(7.4 \%)$ & $5(2.1 \%)$ & $6(2.5 \%)$ & \\
\hline \multicolumn{6}{|l|}{ Nuclear grade } \\
\hline G1 & $86(17.1 \%)$ & $8(29.6 \%)$ & $42(17.6 \%)$ & $36(15.1 \%)$ & \multirow[t]{4}{*}{ C:0.6716 } \\
\hline $\mathrm{G} 2$ & $243(48.2 \%)$ & $14(51.9 \%)$ & $110(46.2 \%)$ & $119(49.8 \%)$ & \\
\hline G3 & $167(33.1 \%)$ & $5(18.5 \%)$ & $81(34.0 \%)$ & $81(33.9 \%)$ & \\
\hline NA/ND & $8(1.6 \%)$ & $0(0.0 \%)$ & $5(2.1 \%)$ & $3(1.3 \%)$ & \\
\hline \multicolumn{6}{|l|}{ ER } \\
\hline Positive & $327(64.9 \%)$ & $15(55.6 \%)$ & $157(66.0 \%)$ & $155(64.9 \%)$ & \multirow[t]{3}{*}{ C:0.7423 } \\
\hline Negative & $163(32.3 \%)$ & $9(33.3 \%)$ & $75(31.5 \%)$ & $79(33.1 \%)$ & \\
\hline NA/ND & $14(2.8 \%)$ & $3(11.1 \%)$ & $6(2.5 \%)$ & $5(2.1 \%)$ & \\
\hline \multicolumn{6}{|l|}{$\operatorname{PgR}$} \\
\hline Positive & $242(48.0 \%)$ & $10(37.0 \%)$ & $113(47.5 \%)$ & $119(49.8 \%)$ & \multirow[t]{3}{*}{ C: 0.5775} \\
\hline Negative & $246(48.8 \%)$ & $14(51.9 \%)$ & $119(50.0 \%)$ & $113(47.3 \%)$ & \\
\hline NA/ND & $10(2.0 \%)$ & $3(11.1 \%)$ & $6(2.5 \%)$ & $1(0.4 \%)$ & \\
\hline \multicolumn{6}{|l|}{$\mathrm{ER} / \mathrm{PgR} *$} \\
\hline Positive & $331(65.7 \%)$ & $15(55.6 \%)$ & $158(66.4 \%)$ & $158(66.1 \%)$ & \multirow[t]{3}{*}{ C:0.8930 } \\
\hline Negative & $159(31.5 \%)$ & $9(33.3 \%)$ & $74(31.1 \%)$ & $76(31.8 \%)$ & \\
\hline NA/ND & $14(2.8 \%)$ & $3(11.1 \%)$ & $6(2.5 \%)$ & $5(2.1 \%)$ & \\
\hline HER $2 *$ & & & & & \\
\hline Positive & $99(19.6 \%)$ & $7(25.9 \%)$ & $44(18.5 \%)$ & $48(20.1 \%)$ & C:0.6576 \\
\hline Negative & $380(75.4 \%)$ & $17(63.0 \%)$ & $183(76.9 \%)$ & $180(75.3 \%)$ & \\
\hline $\mathrm{NA} / \mathrm{ND}$ & $25(5.0 \%)$ & $3(11.1 \%)$ & $11(4.6 \%)$ & $11(4.6 \%)$ & \\
\hline
\end{tabular}

$E R$ estrogen receptor, $F E C$ fluorouracil/epirubicin/cyclophosphamide, $H E R 2$ Human epidermal growth factor receptor 2, $N A$ not available, $N D$ no data, $P g R$ progesterone receptor, $T$ docetaxel alone, $T X$ docetaxle plus capecitabine 


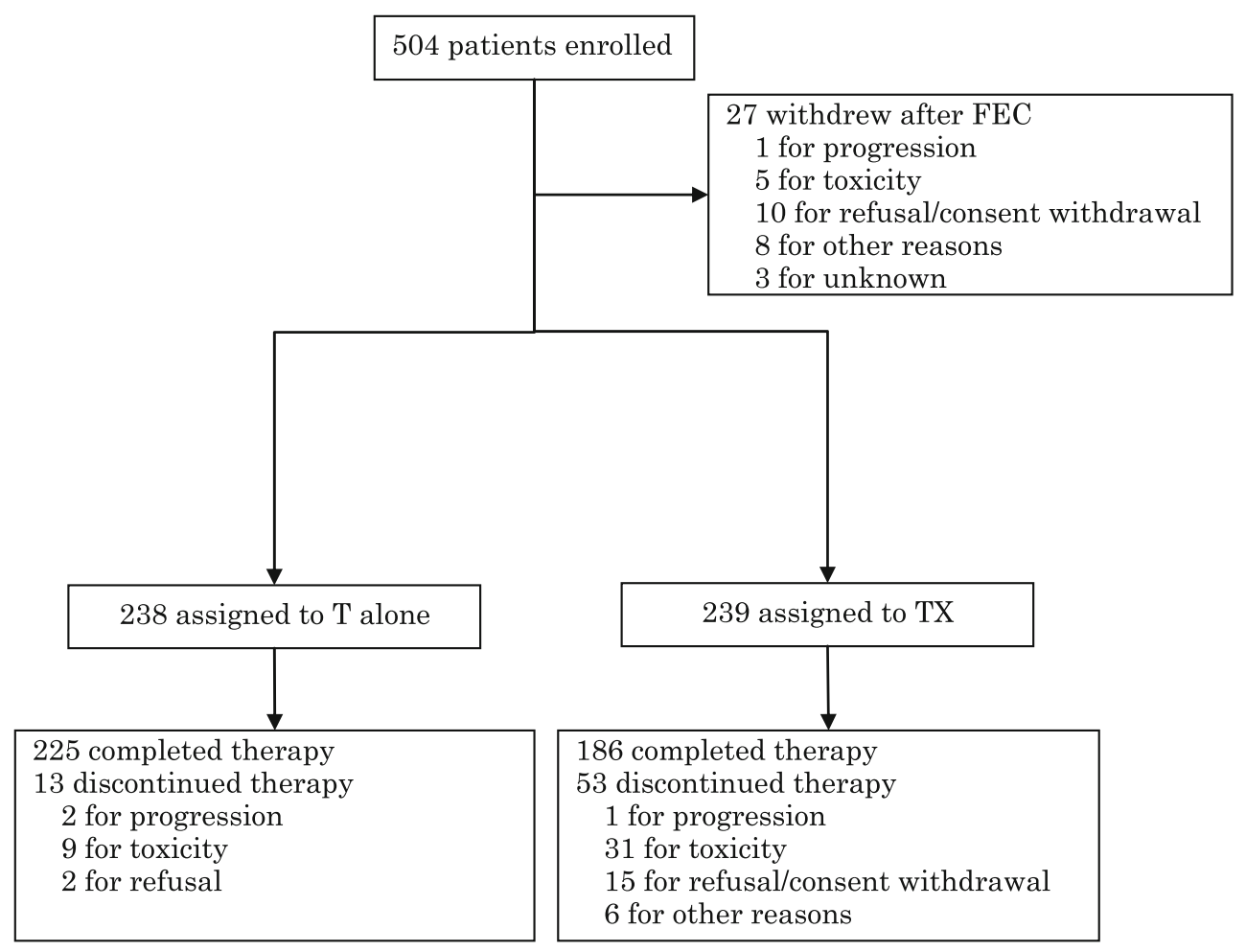

Fig. 1 Study completion. FEC: fluorouracil/epirubicin/cyclophosphamide; T: docetaxel alone; TX: docetaxel plus capecitabine

Table 2 Pathological response by (a) central assessment, (b) central assessment in patients who discontinued or received a reduced dose

\begin{tabular}{|c|c|c|c|c|c|}
\hline & $\begin{array}{l}\text { FEC }(n=27) \\
\%(95 \% \mathrm{CI})\end{array}$ & $\begin{array}{l}\mathrm{TX}(n=239) \\
\%(95 \% \mathrm{CI})\end{array}$ & $\begin{array}{l}\mathrm{T}(n=238) \\
\%(95 \% \mathrm{CI})\end{array}$ & $\begin{array}{l}\text { Difference } \\
(\mathrm{TX}-\mathrm{T})(95 \% \mathrm{CI})\end{array}$ & $p$ value \\
\hline \multicolumn{6}{|l|}{ (a) } \\
\hline $\mathrm{pCR}$ & 7.4 & $23(17.8-28.9)$ & $24.4(19.1-30.3)$ & $-1.4(-9.0$ to 6.3$)$ & 0.7476 \\
\hline pINV & $48.1(28.7-68.1)$ & $72.4(66.3-78.0)$ & $71.4(65.2-77.1)$ & 1 & \\
\hline Missing* & $44.4(25.5-64.7)$ & $4.6(2.3-8.1)$ & $4.2(2.0-6.7)$ & 0.4 & \\
\hline \multicolumn{6}{|l|}{ (b) } \\
\hline $\mathrm{pCR}$ & 7.4 & $23(17.8-28.9)$ & $24.4(19.1-30.3)$ & $-1.4(-9.0$ to 6.3$)$ & 0.7476 \\
\hline With discontinuation & & $(n=12 / 53)$ & $(n=1 / 13)$ & & \\
\hline $\mathrm{pCR}$ & - & $22.6(12.3-36.2)$ & $7.7(0.2-36.0)$ & $14.9(-3.4$ to 33.3$)$ & \\
\hline With dose reduction & & $(n=19 / 79)$ & $(n=2 / 14)$ & & \\
\hline $\mathrm{pCR}$ & - & $24.1(15.1-35.0)$ & $14.3(1.8-42.8)$ & $9.8(-10.8$ to 30.4$)$ & \\
\hline
\end{tabular}

$p C R$ pathological complete response, $p I N V$ pathological presence of invasive tumor, $*$ patients missing post-baseline mainly due to discontinuation as a result of toxicity, $C I$ confidence interval, FEC 5-fluorouracil-epirubicin-cyclophosphamide, $T X$ docetaxel plus capecitabine, $T$ docetaxel alone

Table 2a). However, we observed an interesting trend in the subset of patients who had discontinued treatment or received a $25 \%$ dose reduction. Despite treatment withdrawal, $12 / 53$ in the docetaxel/capecitabine group and 1/13 in the docetaxel group achieved a pCR with rates of 22.6 and $7.7 \%$, respectively. A similar trend was observed in the $33.1 \%(79 / 239)$ and $5.9 \%(14 / 238)$ who received a $25 \%$ dose reduction and achieved pCR rates of $24.1 \%$ $(19 / 79)$ and $14.3 \%$ (2/14), respectively (Table 2b).
Although not statistically significant, pCR rates were higher in the docetaxel/capecitabine group in comparison to the docetaxel group in this subpopulation.

Disease-free and overall survival

After a median 4.5-year follow-up, the 3-year DFS was estimated at $92.7 \%$ in the docetaxel/capecitabine group and $90.7 \%$ in the docetaxel group. Four patients were 


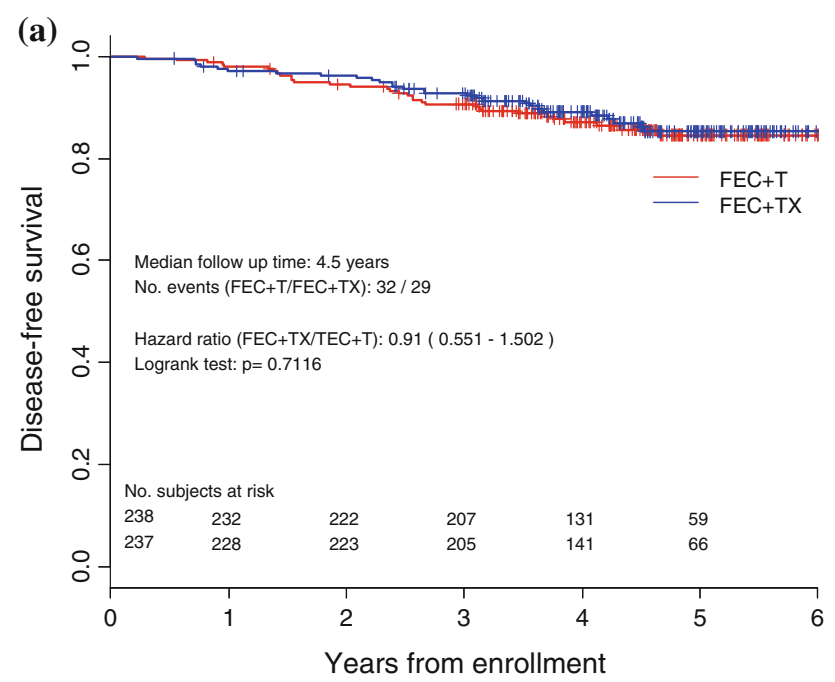

(b)

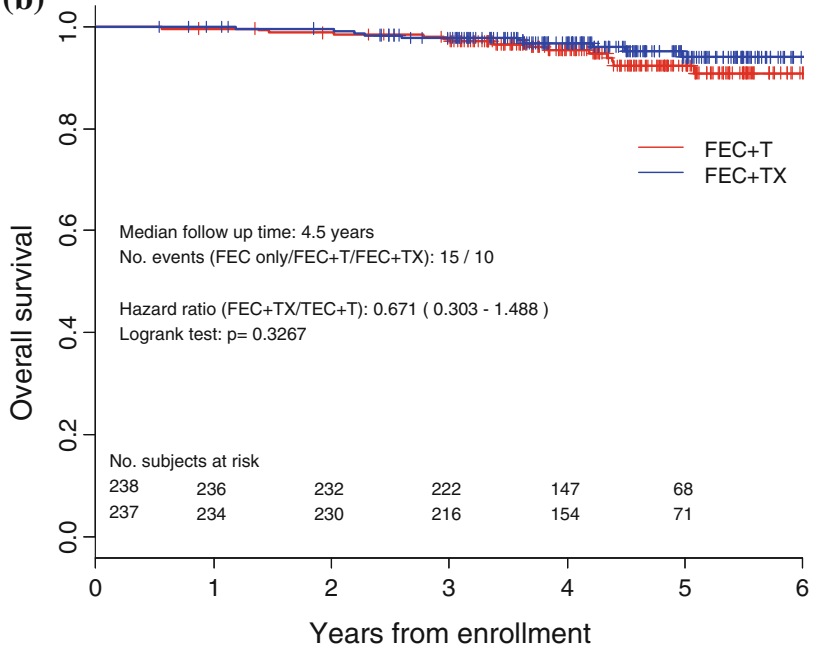

Fig. 2 a Disease-free survival. b Overall survival. FEC: fluorouracil/ epirubicin/cyclophosphamide; T: docetaxel alone; TX: docetaxel plus capecitabine

excluded from the ITT population due to missing data. A total of 29 events occurred in the docetaxel/capecitabine group and 32 in the docetaxel group, with a HR of 0.910 (95\% CI 0.551-1.502; Fig. 2a). During follow-up, 10 deaths occurred in the docetaxel/capecitabine group and 15 in the docetaxel group, with a point of estimate HR of 0.671 (95\% CI 0.303-1.488; Fig. 2b).

Predictive factor analyses for pathological response and survival status

Subpopulation analysis for pathological response showed no significant difference between treatment groups (data not shown). To identify predictive factors for pathological response using age and $\mathrm{Ki} 67$ as continuous variables, an overlapping subpopulation of 84 patients was constructed and analyzed using the STEPP method. Although no

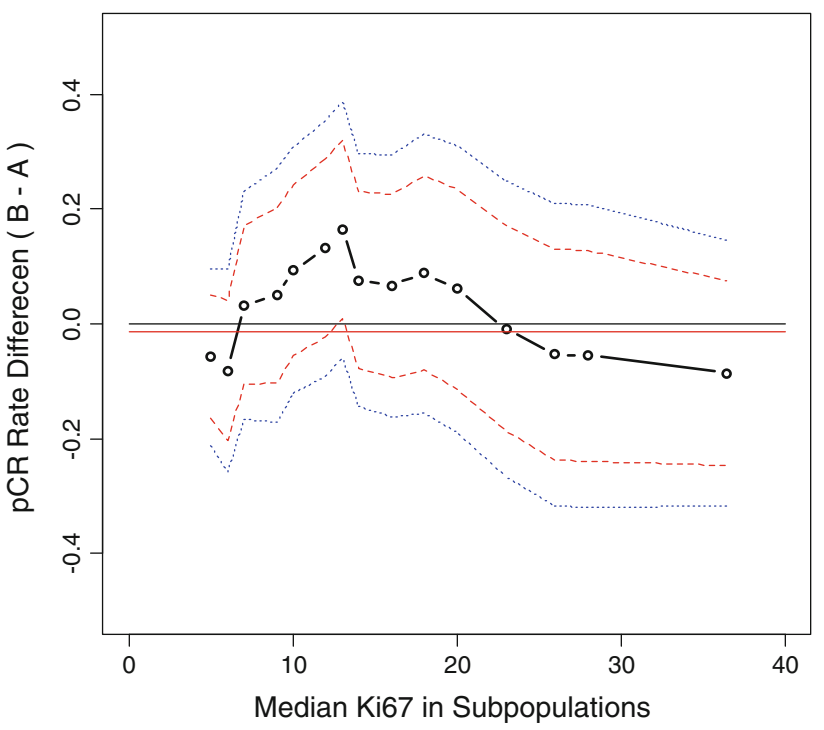

Fig. 3 STEPP analysis of the treatment effect of docetaxel/capecitabine compared with single-agent docetaxel as measured by pCR. Values $>0$ suggested that the combination regimen was better; $<0$ indicated that single-agent docetaxel was better. Difference in pCR is shown (dashed black lines) with corresponding $95 \%$ CI (dashed red lines) and corresponding $95 \%$ confidence band (dashed blue lines). Overall difference in pCR (solid horizontal red line) is shown

statistical significance was achieved, STEPP analysis indicated a trend in favor of improved pCR rate in patients with mid-range of Ki67LI (10-20\%) following docetaxel/ capecitabine compared with docetaxel alone (Fig. 3). To further investigate the predictive value of Ki67 relative to $\mathrm{pCR}$, univariate and multiple logistic regression models were fitted to calculate the odds ratio (OR) and $95 \% \mathrm{CI}$ for each risk factor.

Univariate analysis showed that nuclear grading, ER and/or PgR status, HER2 status, baseline Ki67 and TP-SI were all strongly associated with pCR (Table 3a). Multivariate analysis was performed using the predictive variables identified in the univariate analysis. To evaluate the effect of Ki67, a multivariate logistic regression analysis was undertaken in 410 patients with available baseline data for nuclear grading, ER and/or PgR, HER2, and Ki67. In the first model, all of these factors continued to be $15 \%$ significant predictors for $\mathrm{pCR}$. In the final model, pretreatment levels of Ki67 proved to be a predictive factor for pCR, with an OR of 1.031 (95\% CI 1.014-1.048; $p=0.0004)$. Using this model, the random cross-validated sensitivity and specificity were 83.3 and $63.4 \%$, respectively (Table 3b).

Predictive factors for DFS were analyzed using a multiple Cox model in a landmark analysis (Online Resource). When pCR and postKi67 were included in the final model, tumor stage (I, IIa/III: HR 0.144, $95 \%$ CI 0.051-0.404; IIb/III: HR 0,264, $95 \%$ CI 0.107-0.651; $p=0.0006$ ), cancer cell TP status (continuous variables: HR 0.966, 
Table 3 Prediction of pCR using (a) simple logistic regression model, (b) multiple logistic regression model with Ki67

\begin{tabular}{|c|c|c|c|c|c|c|}
\hline Factor & \# pat & & \# res & OR & $95 \% \mathrm{CI}$ & $p$ value \\
\hline \multicolumn{7}{|l|}{ (a) } \\
\hline \multicolumn{7}{|l|}{ Age } \\
\hline$\leq 49$ & 248 & & 56 & 0.880 & $0.577-1.343$ & 0.5534 \\
\hline$\geq 50$ & 229 & & 57 & 1 & & \\
\hline \multicolumn{7}{|l|}{ Initial tumor size } \\
\hline$\leq 2.0$ & 29 & & 7 & 1.047 & $0.409-2.682$ & 0.9919 \\
\hline $2.1-4.0$ & 315 & & 75 & 1.028 & $0.637-1.659$ & \\
\hline$\geq 4.1$ & 133 & & 31 & 1 & & \\
\hline \multicolumn{7}{|l|}{ Axillary lymph node } \\
\hline Positive & 268 & & 62 & 0.932 & $0.610-1.426$ & 0.7467 \\
\hline Negative & 209 & & 51 & 1 & & \\
\hline \multicolumn{7}{|l|}{ Menopausal status } \\
\hline Pre & 266 & & 60 & 0.868 & $0.568-1.326$ & 0.5135 \\
\hline Post & 211 & & 53 & & & \\
\hline \multicolumn{7}{|l|}{ Stage } \\
\hline I/IIa & 54 & & 211 & 1.849 & $0.818-4.179$ & 0.3355 \\
\hline IIb & 51 & & 215 & 1.671 & $0.738-3.786$ & \\
\hline III & 8 & & 51 & 1 & & \\
\hline \multicolumn{7}{|l|}{ Nuclear grading } \\
\hline G1 & 78 & & 9 & 0.240 & $0.112-0.517$ & $<.0001$ \\
\hline $\mathrm{G} 2$ & 229 & & 46 & 0.463 & $0.293-0.731$ & \\
\hline G3 & 162 & & 57 & 1 & & \\
\hline ER and/or PgR & & & & & & \\
\hline Positive & 327 & & 58 & 0.265 & $0.167-0.422$ & $<.0001$ \\
\hline Negative & 116 & & 52 & 1 & & \\
\hline HER2 & & & & & & \\
\hline Positive & 62 & & 33 & 4.552 & $2.604-7.958$ & $<.0001$ \\
\hline Negative & 380 & & 76 & 1 & & \\
\hline Baseline of Ki67 (\%) & & & & & & \\
\hline$\geq 10$ & 299 & & 95 & 4.572 & $2.348-8.903$ & $<.0001$ \\
\hline$<10$ & 119 & & 11 & 1 & & \\
\hline Continuous & 418 & & & 1.043 & $1.027-1.059$ & $<.0001$ \\
\hline TP-CI & & & & & & \\
\hline $1+, 2+, 3+$ & 282 & & 73 & 1.715 & $0.851-3.456$ & 0.1316 \\
\hline 0 & 65 & & 11 & 1 & & \\
\hline $2+, 3+$ & 119 & & 33 & 1.332 & $0.801-2.213$ & 0.2690 \\
\hline $0,1+$ & 228 & & 51 & 1 & & \\
\hline TP-SI & & & & & & \\
\hline $1+, 2+, 3+$ & 324 & & 84 & 4.025 & $0.929-17.438$ & 0.0627 \\
\hline 0 & 25 & & 2 & 1 & & \\
\hline $2+, 3+$ & 197 & & 59 & 1.979 & $1.182-3.315$ & 0.0095 \\
\hline $0,1+$ & 152 & & 27 & 1 & & \\
\hline OR & $95 \% \mathrm{CI}$ & $p$ value & Ser & city & ROC $(95 \%$ CI $)$ & al model \\
\hline (b) & & & & & & \\
\hline Grading & & & & & & \\
\hline 0.312 & $0.129-0.756$ & 0.0027 & $\mathrm{Ra}$ & & Apparent & \\
\hline 0.461 & $0.274-0.773$ & & Ser & & 0.7510 & \\
\hline
\end{tabular}


Table 3 continued

\begin{tabular}{|c|c|c|c|c|c|c|}
\hline & OR & $95 \% \mathrm{CI}$ & $p$ value & Sensitivity specificity & ROC $(95 \% \mathrm{CI})$ & Contrast with final model \\
\hline 3 & 1 & & & $(0.6034,0.8958)$ & $(0.6999,0.8021)$ & \\
\hline \multicolumn{7}{|l|}{ ER and/or PgR } \\
\hline Positive & 0.384 & $0.230-0.642$ & 0.0003 & & & \\
\hline Negative & 1 & & & Spe: 0.6097 & Random cv & \\
\hline \multicolumn{7}{|l|}{ HER2 } \\
\hline Positive & 3.816 & $2.056-7.081$ & $<.0001$ & $(0.5517,0.7391)$ & 0.7353 & \\
\hline Negative & 1 & & & & $(0.6664,0.7901)$ & \\
\hline \multicolumn{7}{|l|}{ Grading } \\
\hline 1 & 0.402 & $0.163-0.991$ & 0.00281 & Random cv & Apparent & Apparent \\
\hline 2 & 0.536 & $0.316-0.909$ & & Sen: 0.8000 & 0.7657 & 0.0147 \\
\hline 3 & 1 & & & $(0.6599,0.8889)$ & $(0.7172,0.8143)$ & $(-0.0055,0.0350)$ \\
\hline \multicolumn{7}{|l|}{ ER and/or PgR } \\
\hline Positive & 00.413 & $0.247-0.692$ & 0.0008 & & & \\
\hline Negative & 1 & & & Spe: 0.6458 & & \\
\hline \multicolumn{7}{|l|}{ HER2 } \\
\hline Positive & 3.522 & $1.890-6.563$ & $<.0001$ & $(0.5792,0.7452)$ & Random cv & Random cv \\
\hline Negative & & & & & 0.7489 & 0.0168 \\
\hline \multicolumn{7}{|l|}{$\operatorname{Ki67}(\%)$} \\
\hline$\geq 10$ & 2.718 & $1.331-5.549$ & 0.0061 & & $(0.6827,0.7986)$ & $(-0.0303,0.041)$ \\
\hline$<10$ & 1 & & & & & \\
\hline \multicolumn{7}{|l|}{ Grading } \\
\hline 1 & 0.418 & $0.169-1.035$ & 0.0298 & Random cv & Apparent & Apparent \\
\hline 2 & 0.530 & $0.312-0.900$ & & Sen: 0.8333 & 0.7774 & 0.0264 \\
\hline 3 & 1 & & & $(0.6735,0.9400)$ & $(0.7289,0.8259)$ & $(0.0015,0.0513)$ \\
\hline \multicolumn{7}{|l|}{ ER and/or PgR } \\
\hline Positive & 0.447 & $0.265-0.754$ & 0.0026 & & & \\
\hline Negative & 1 & & & Spe: 0.6344 & Random cv & Random cv \\
\hline \multicolumn{7}{|l|}{ HER2 } \\
\hline Positive & 3.794 & $2.038-7.065$ & $<0.0001$ & $(0.5063,0.7713)$ & 0.7607 & 0.0274 \\
\hline Negative & 1 & & & & $(0.6993,0.8099)$ & $(-0.0175,0.0596)$ \\
\hline Ki67 (continuous) & 1.031 & $1.014-1.048$ & 0.0004 & & & \\
\hline
\end{tabular}

\#pat number of patients, \#res number of responders, CI confidence interval, ER estrogen receptor, HER2 human epidermal growth factor receptor 2, $O R$ odds ratio, $P g R$ progesterone receptor, $T P$ - $C I$ thymidine phosphorylase, interstitial, $T P$-SI thymidine phosphorylase, stromal

$E R$ estrogen receptor, $H E R 2$ human epidermal growth factor receptor 2, $P g R$ progesterone receptor, $O R$ odds ratio

$95 \%$ CI $0.941-0.993 ; p=0.0125)$, pCR, and post-treatment Ki67LI (pCR/Ki67 <10/ $\geq 10$ : HR 0.269, $95 \%$ CI $0.110-0.655 ; p=0.0038)$ were all significantly associated with DFS (Table 4).

\section{Safety profile}

The frequency of major adverse events ( $\geq$ grade 3 ) of docetaxel/capecitabine and docetaxel group were as follows: leukopenia (36 and $34 \%$, respectively), neutropenia (38 and $34 \%$, respectively), febrile neutropenia ( 8 and $5 \%$, respectively), and hand-foot syndrome (15 and $2 \%$, respectively). Docetaxel/capecitabine was associated with more capecitabine-related toxicity, including hand-foot syndrome, nausea, mucositis, and increased alanine aminotransferase. Six serious adverse events were reported for 3 patients in the docetaxel/capecitabine group (pneumonitic cough, muscle pain, neutropenia fever) and 3 patients in the docetaxel group (suicide, loss of eyesight of left eye, hematological toxicity). The event of suicide in the docetaxel alone group occurred after completion of treatment and was considered as unrelated to study treatment.

\section{Discussion}

We have presented results from a randomized study comparing preoperative capecitabine/docetaxel with docetaxel 
Table 4 Hazard ratio for disease-free survival using a multiple cox model in landmark analysis

\begin{tabular}{llll}
\hline Factors & HR & $(95 \% \mathrm{CI})$ & $p$ value \\
\hline
\end{tabular}

The final model

Stage

I/IIa

$\mathrm{IIb}$

0.160

0.059-0.436

0.390

0.170-0.893

III

1

ER and/or PgR

Positive

Negative

0.468

0.974

0.235-0.932

0.953-0.996

TP-CP

Continuous

1

Extended model 1

Stage

I/IIa

$\mathrm{IIb}$

III

0.170
0.360
1

$0.065-0.444$

0.165-0.787

ER and/or PgR

Positive

Negative

TP-CP

Continuous

pCR

Responder

Nonresponder

Extended model 2

Stage

IIIIa

ER and/or PgR

$$
\text { Positive }
$$

Negative

TP-CP

Continuous

0.327

1

$0.160-0.670$

0.0023

0.975

0.191

0.954-0.997

$0.052-0.696$

Treatment

Completion

Reduction

Discontinuation

Extended model 3

Stage

$\begin{array}{lll}\text { I/IIa } & 0.134 & 0.051-0.350 \\ \text { IIb } & 0.309 & 0.135-0.706 \\ \text { III } & 1 & \end{array}$

ER and/or PgR

$\begin{array}{llll}\text { Positive } & 0.439 & 0.220-0.878 & 0.0200 \\ \text { Negative } & 1 & & \\ \begin{array}{l}\text { TP-CP } \\ \text { Continuous }\end{array} & 0.974 & 0.953-0.996 & 0.0183\end{array}$

0.051-0.349

0.0002

0.308

0.134-0.706

1

0.441

$0.221-0.878$

0.0198

1

0.953-0.996

0.0199

0.209-1.917

0.3560

1.125

$0.339-3.729$

1
Table 4 continued

\begin{tabular}{llll}
\hline Factors & HR & $(95 \%$ CI $)$ & $p$ value \\
\hline $\begin{array}{l}\text { Treatment } \\
\text { Completion }\end{array}$ & 0.584 & $0.278-1.226$ & 0.1554 \\
$\quad$ Otherwise & 1 & & \\
Extended model 4 & & & \\
Stage & & & 0.0006 \\
I/IIa & 0.153 & $0.056-0.419$ & \\
IIb & 0.279 & $0.116-0.673$ & \\
III & 1 & & 0.2433 \\
ER and/or PgR & & & \\
Positive & 0.577 & $0.229-1.454$ & \\
Negative & 1 & & 0.0144 \\
TP-CP & & & \\
Continuous & 0.967 & $0.941-0.993$ & \\
p CR \& PostKi67 & & & \\
Responder & 0.137 & $0.034-0.549$ & \\
PostKi67 $<10$ & 0.388 & $0.143-1.052$ & \\
PostKi67 $\geqq 10$ & 1 & &
\end{tabular}

Extended model 5

Stage

$\begin{array}{llll}\text { I/IIa } & 0.144 & 0.051-0.404 & 0.0006 \\ \text { IIb } & 0.264 & 0.107-0.651 & \\ \text { III } & 1 & & \end{array}$

ER and/or PgR

Positive

Negative

TP-CP

Continuous

pCR \& PostKi67

Responder

PostKi67 $<10$

PostKi67 $\geqq 10$

Extended model 5

Stage

$\begin{array}{llll}\text { I/IIa } & 0.200 & 0.072-0.561 & 0.0031 \\ \text { IIb } & 0.264 & 0.103-0.676 & \\ \text { III } & 1 & & \\ \text { ER and/or PgR } & & & \\ \text { Positive } & 0.385 & 0.152-0.977 & 0.0445 \\ \text { Negative } & 1 & & \\ \text { TP-CP } & & & 0.0301 \\ \text { Continuous } & 0.970 & 0.943-0.997 & \end{array}$

$E R$ estrogen receptor, $H E R 2$ human epidermal growth factor receptor 2, $P g R$ progesterone receptor, $O R$ odds ratio, $T P-C P$ thymidine phosphorylase, plasma

alone after FEC in early-stage breast cancer, and have identified Ki67 as a predictive biomarker that may be used to identify patients likely to respond to this neoadjuvant regimen. 
In contrast to previous reports, we observed no difference in the $\mathrm{pCR}$ rate between the docetaxel/capecitabine and the docetaxel group. Our observation was similar to that from the GeparQuattro study, in which docetaxel/ capecitabine did not improve pCR rate in comparison to docetaxel after epirubicin/cyclophosphamide treatment in the neoadjuvant setting [21]. Although a $16 \%$ pCR rate was expected in the docetaxel group based on previous observations [5], the pCR rate in our study was higher (24\%). The variation in clinical outcome may be attributed to the currently limited means with which to select patient subpopulations most likely to respond to a given treatment regimen.

The docetaxel/capecitabine regimen was less well tolerated than docetaxel alone, with withdrawal rates of 22.2 and $5.5 \%$ and dose reduction rates of 33.1 and $5.9 \%$, respectively. Despite treatment withdrawals and dose reductions, achievement of higher pCR rates in the docetaxel/capecitabine group in comparison to the docetaxel group in this subpopulation suggests that dose reduction does not negatively impact capecitabine efficacy. Our data confirms a similar observation in a MBC study, which reported no significant effect on efficacy when dose reduction occurred in 65 and $36 \%$ of patients receiving the docetaxel/capecitabine regimen and docetaxel alone, respectively. However, an increased risk of disease progression was seen in patients with a dose reduction to $50 \%$ of the starting dose in the docetaxel group (HR 1.91) [15]. As reported by other groups [22], our data demonstrate that the capecitabine dose can be reduced to minimize adverse effects without compromising efficacy. It was, however, interesting to observe that patients who discontinued or received a dose reduction in the docetaxel/capecitabine group achieved a higher pCR compared with the docetaxel alone group, while there was no difference in pCR between both groups in patients that completed the study at the original dose. Although the reason for this observation is unclear, the observation that the relative dose intensity for docetaxel was significantly lower in the combination arm compared with the single agent docetaxel arm may at least in part, account for the lack of difference in pCR. In addition, levels of toxicity may have had an impact on drug delivery and thus, pCR.

In addition to comparing the efficacy of neoadjuvant docetaxel/capecitabine with docetaxel alone, our study also sought to identify biomarkers that can identify patients likely to respond to treatment with docetaxel/capecitabine in early-stage breast cancer. Previously identified biomarkers, such as nuclear grading, ER and/or PgR status, HER2 status and Ki67, correlated with pCR in our study, as in other published studies [23]. Of particular interest was pre-treatment Ki67LI, which had a strong correlation with pCR and added to the predictive value of the multivariate logistic regression model. Indeed, data from several other studies suggest that high Ki67 levels in breast cancer are a predictive factor for pCR rate [5, 24-27]. This effect was present in our study, as patients with $\geq 10 \%$ pre-treatment Ki67LI achieved a higher pCR rate in both the docetaxel/ capecitabine $(32.6 \%)$ and docetaxel alone $(31 \%)$ groups, in comparison to patients with $<10 \%$ pre-treatment Ki67LI (pCR rates 6.5, $12.3 \%$, respectively). These findings support the suggestion that detection of pre-treatment Ki67LI could identify patients most likely to benefit from neoadjuvant chemotherapy. The prognostic value of Ki67 was also confirmed in our study, as post-treatment Ki67LI and PCR were significantly associated with DFS using a multiple Cox model in a landmark analysis. Thus, prognostic and predictive value was detected for Ki67, showing it to be a feasible marker for development of individualized treatment options for early-stage breast cancer patients.

To our knowledge, this is the first multicenter randomized study showing that assessment of pre- and post-treatment Ki67 may be a useful tool in predicting pCR and DFS with neoadjuvant docetaxel treatment with or without capecitabine in patients with early-stage breast cancer. Although further studies are required, our data suggests that the routine detection of the Ki67 proliferation marker in early-stage breast cancer could be a useful prognostic tool for the identification of patients most likely respond to preoperative docetaxel with or without capecitabine. As such, in addition to the current leading parameters $(\mathrm{ER}, \mathrm{PgR}$, and HER2 status), we propose that Ki67 should be included in the list of required routine biological markers that are used to define treatment recommendations in patients with early-stage breast cancer. Indeed, detection of predictive biomarkers prior to chemotherapy is likely to prove to be of the greatest advantage for neoadjuvant chemotherapy.

Acknowledgments This study was made possible by the generous support and supply of knowledge by Product Research Department, Kamakura Research Laboratories, Chugai Pharmaceutical Co., Ltd. and Sanofi K.K., Japan. This work was supported by an unconditional grant from Sanofi K.K., Japan.

Conflict of interest The authors have declared no conflicts of interest.

Open Access This article is distributed under the terms of the Creative Commons Attribution Noncommercial License which permits any noncommercial use, distribution, and reproduction in any medium, provided the original author(s) and the source are credited.

\section{References}

1. Wolmark N, Wang J, Mamounas E et al (2001) Preoperative chemotherapy in patients with operable breast cancer: 9-year results from National Surgical Adjuvant Breast and Bowel Project B-18. J Natl Cancer Inst Monogr 30:96-102 
2. Rastogi P, Anderson SJ, Bear HD et al (2008) Preoperative chemotherapy: updates of National Surgical Adjuvant Breast and Bowel Project Protocols B-18 and B-27. J Clin Oncol 26(5):778-785

3. Rouzier R, Perou C, Symmans W et al (2005) Breast cancer molecular subtypes respond differently to preoperative chemotherapy. Clin Cancer Res 11:5678-5685

4. Nolen B, Marks KJ, Ta'san S et al (2008) Serum biomarker profiles and response to neoadjuvant chemotherapy for locally advanced breast cancer. Breast Cancer Res 10:R45

5. Fasching PA, Heusinger K, Haeberle L et al (2011) Ki67, chemotherapy response, and prognosis in breast cancer patients receiving neoadjuvant treatment. BMC Cancer 11:486

6. Bear HD, Anderson S, Smith RE et al (2006) Sequential preoperative or postoperative docetaxel added to preoperative doxorubicin plus cyclophosphamide for operable breast cancer: National Surgical Adjuvant Breast and Bowel Project Protocol B-27. J Clin Oncol 24:2019-2027

7. Toi M, Nakamura S, Kuroi K et al (2008) Phase II study of preoperative sequential FEC and docetaxel predicts of pathological response and disease free survival. Breast Cancer Res Treat 110:531-539

8. Sawada N, Ishikawa T, Fukase Y et al (1998) Induction of thymidine phosphorylase activity and enhancement of capecitabine efficacy by taxol/taxotere in human cancer xenografts. Clin Cancer Res 4:1013-1019

9. Miwa M, Ura M, Nishida M et al (1998) Design of a novel oral fluoropyrimidine carbamate, capecitabine, which generates 5 -fluorouracil selectively in tumours by enzymes concentrated in human liver and cancer tissue. Eur J Cancer 34:1274-1281

10. Blum JL, Jones SE, Buzdar AU et al (1999) Multicenter phase II study of capecitabine in paclitaxel-refractory metastatic breast cancer. J Clin Oncol 17:485-493

11. Blum JL, Dieras V, Lo Russo PM et al (2001) Multicenter, phase II study of capecitabine in taxane-pretreated metastatic breast carcinoma patients. Cancer 92:1759-1768

12. Jinno $H$, Sakata M, Hayashida $T$ et al (2010) A phase II trial of capecitabine and docetaxel followed by 5-fluorouracil/epirubicin/ cyclophosphamide (FEC) as preoperative treatment in women with stage II/III breast cancer. Ann Oncol 21:1262-1266

13. Natoli C, Cianchetti E, Tinari N et al (2007) A phase II study of dose-dense epirubicin plus cyclophosphamide followed by docetaxel plus capecitabine and pegfilgrastim support as preoperative therapy for patients with stage II, IIIA breast cancer. Ann Oncol 18:1015-1020

14. Lebowitz PF, Eng-Wong J, Swain SM et al (2004) A phase II trial of neoadjuvant docetaxel and capecitabine for locally advanced breast cancer. Clin Cancer Res 10:6764-6769
15. O'Shaughnessy J, Miles D, Vukelja S et al (2002) Superior survival with capecitabine plus docetaxel combination therapy in anthracycline-pretreated patients with advanced breast cancer: phase III trial results. J Clin Oncol 20:2812-2823

16. Bouzubar N, Walker KJ, Griffiths K et al (1989) Ki67 immunostaining in primary breast cancer: pathological and clinical associations. Br J Cancer 59:943-947

17. Mikami Y, Ueno T, Yoshimura K, Tsuda H, Kurosimi M, Masuda $\mathrm{S}$ et al. (2013) Inter-observer concordance of Ki67 labeling index in breast cancer. Japan Breast Cancer Research Group (JBCRG) Ki67 Ring Study. Cancer Sci. doi:10.1111/cas.12245

18. Stefanou D, Batistatou A, Nonni A, Arkoumani E, Agnantis NJ (2004) p63 expression in benign and malignant breast lesions. Histol Histopathol 19(2):465-471

19. Dewar R, Fadare O, Gilmore H, Gown AM (2011) Best practices in diagnostic immunohistochemistry: myoepithelial markers in breast pathology. Arch Pathol Lab Med 135(4):422-429

20. Werling RW, Hwang H, Yaziji H, Gown AM (2003) Immunohistochemical distinction of invasive from noninvasive breast lesions: a comparative study of p63 versus calponin and smooth muscle myosin heavy chain. Am J Surg Pathol 27(1):82-90

21. von Minckwitz G, Rezai M, Loibl S et al (2010) Capecitabine in addition to anthracycline- and taxane-based neoadjuvant treatment in patients with primary breast cancer: phase III GeparQuattro Study. J Clin Oncol 28(12):2015-2023

22. Leonard R, O'Shaughnessy J, Vukelja S et al (2006) Detailed analysis of a randomized phase III trial: can the tolerability of capecitabine plus docetaxel be improved without compromising its survival advantage? Ann Oncol 17:1379-1385

23. Caudle AS, Ganzalez-Angulo AM, Hunt KL et al (2010) Predictors of tumor progression during neoadjuvant chemotherapy in breast cancer. J Clin Oncol 28(11):1821-1828

24. Houssami N, Macaskill P, von Minckwitz G, Marinovich ML, Mamounas E et al (2012) Meta-analysis of the association of breast cancer subtype and pathologic complete response to neoadjuvant chemotherapy. Eur J Cancer 48(18):3342-3354

25. Yerushalmi R, Woods R, Ravdin PM et al (2010) Ki67 in breast cancer: prognostic and predictive potential. Lancet Oncol 11:174-183

26. Zhang GC, Qian XK, Guo ZB et al (2012) Pre-treatment hormal receptor status and Ki67 index predict pathological complete response to neoadjuvant trastuzumab/taxanes but not disease-free survival in HER2-positive breast cancer patients. Med Oncol 29(5):3222-3231

27. Luporsi E, Andre F, Spyratos F et al (2012) Ki-67: level of evidence and methodoligical considerations for its role in the clinical management of breast cancer: analytical and critical review. Breast Cancer Res Treat 132(3):895-915 